dealt with for this value of $\rho$ by using the appropriate degree of telescopic magnification or demagnification of the beam aperture.) To determine the instrumental profile and finesse more precisely a computer wavefront calculation is being carried out, and the effects of coherent and incoherent illumination are also being studied.

The interferogram of Fig. 2 was photographed by $\mathbf{M r}$ M. E. Engwell and the calibration curve was derived by Mr A. Durrant. This work is supported by the Science Research Council.

Department of Physies,

\section{J. Bradley}

\section{Queen's University of Belfast.}

Received February 10; revised May 9, 1967.

${ }^{1}$ Connes, P., J. Phys., 19, 262 (1958).

' Jackson, D. A., Proc. Roy. Soc., A., 263, 289 (1961).

${ }^{3}$ Hanes, G. R., and Stoicheff, B. P., Nature, 195, 587 (1962).

${ }^{4}$ Bradley, D. J., Engwell, M. S., MeCullough, A. W., Magyar, G., and Richardson, M. (', App. Phys. Lett., 9, 150 (1966).

${ }^{5}$ Bradley, D. J., Magyar, G., and Richardson, M. C., Nature, 212, 63 (1966).

${ }^{6}$ Nienhuis, K., thesis, Univ. Gröningen (1948).

" Zernike, F., Proc. Phys. Soc. Lond., 61, 158 (1948).

${ }^{8}$ Bradley, D. J., Optica Acta, 9, 365 (1962).

${ }^{9}$ Bradley, D. J., and Mitchell, C. (to be published).

${ }^{10}$ Bradley, D. J., Bates, B., Juulman, C. O. L., and Majumdar, S., App, Optics, 3, 1461 (1964).

\section{Relativistic Equations of State}

WE offer a new approach to an old 1 question: "Given a material medium with its equation of state, what conditions must be satisfied by that equation of state if the existence of such matter is to be consistent with the principles of special relativity ?" Our object is to determine one such condition. Our approach has the advantage that it is not necessary to consider the detailed structure of the matter, but it has the disadvantage that it is not applicable in its present form to the most interesting case- that of nuclear matter at high densities.

The two traditional approaches to this question may be summarized as follows:

(1) It has been claimed ${ }^{2}$ that the assumption of causality* implies that $\mathrm{d} p / \mathrm{d} \rho \leq 1$, where $p$ is the pressure and $\rho$ is the mass density of a fluid. (We have set $c=1$.) This inequality may be shown to hold rigorously for a perfect fluid characterized only by the two parameters $p$ and $\rho$, with conserved stress-energy $T_{\alpha \beta}=(p+\rho) \xi_{\alpha} \xi_{\beta}+p \quad g_{\alpha \beta}$. (Here, $\xi_{\alpha}$ is the unit 4 -velocity of a fluid element at each point.) Real matter, however, is more complicated. One must also consider temperature, composition and possibly other variables-the equation of state may, for example, depend on the past history of the matter. When these detailed properties of the matter are taken into account, no simple conclusion such as $\mathrm{d} p / \mathrm{d} \rho \leq 1$ can be drawn.

(2) It has been claimed ${ }^{3}$ that the "reasonable physical assumption" that the behaviour of high-energy particles is similar to that of photons implies that $p \leq(1 / 3) p$ for all matter. This inequality is in fact satisfied for the case of a relativistic gas of non-interacting particles. ${ }^{4}$. There is, however, no compelling reason why the equation of state for a gas of non-interacting particles should approximate that for matter (see, for example, the discussion of Zeldovich ${ }^{5}$ ).

Our approach may be illustrated by a simple example based on a spring characterized by the following three parameters: unstressed length $=L$; mass per unit length $=\rho$; spring constant $\times$ length $=p$.

We assume energy conservation and causality. Accelerate the spring to a velocity $v<1$ so that, during the acceleration process, the length of the spring in its own rest frame is kept constant. We wish to entrap the spring

${ }^{*}$ This implies that if initial data for any system are given on a space-like surface $S$, the state of the system at any point $P$ of space-time is uniquely determined by the data on the intersection of $S$ with the interior of the backward light cone of $P$. in a cylindrical container of length $b$, closed at the rear and with a sliding door at the front. At the moment the leading end of the spring strikes the rear of the container, a light signal is sent along the spring. If the length $b$ is chosen to be $L \frac{\sqrt{1-v^{2}}}{1+v}$, then this signal reaches the trailing end of the spring just as this end enters the container. The sliding door is then shut. Thus the assumption of causality, which entails that the information that the leading end of the spring has struck the container does not reach the trailing end of the spring faster than light, determines the size of the container necessary to entrap the spring. After the spring has settled down and cooled off, the container is opened and the spring is allowed to expand. The energy released in this expansion is carefully collected. The requirement that the energy necessary to accelerate the spring should be greater than or equal to that released by the expansion is

$$
\rho\left(\frac{1}{\sqrt{1-v^{2}}}-1\right) \geq \frac{1}{2} p\left(1-\frac{\sqrt{1-v^{2}}}{1+v}\right)^{2}
$$

This inequality will hold for all $v<1$ if, and only if,

$$
\rho \geq p
$$

One would hope to apply a similar argument to nuclear matter at high densities. There is, however, a serious difficulty: it is necessary to introduce a second container to hold the matter under high pressure during the acceleration process. In order to obtain the inequality $p \leq p$, it is now necessary to find a gedanken procedure to recover the energy involved in accelerating this second container.

\section{R. P. Geboch}

D. J. HEGYI

Palmer Physical Laboratory,

Princeton, New Jersey.

Received June 19, 1967.

1 Pauli, W., Theory of Relativity, 134 (Pergamon Press, London, 1958).

2 Landau, L. D., and Lifshitz, E. M., Fluid Mechanics, 502 (Pergamon Press, London, 1959). Wheeler, J. A., in Relativity, Groups, and Topology, (edit. by DeWitt, B., and DeWitt, C.), 322 (Gordon and Breach, New York, 1964)

${ }^{3}$ Landau, I. D., and Lifshit, E. M., The Classical Theory of Fields, 99 (Pergamon Press, London, 1962).

Synge, J. L., The Relativistic Gas, 36 (North Holland Publ. Co., Amsterdam, 1957).

Zeldovich, Ya. B,, J. Exp. and Theo. Phys. (USSR), 41, 1609 (1961) (Soviet Physics JETP, 14, $1143(1962))$.

\section{States in Neon-2I near $3 \mathrm{MeV}$}

Properties of low lying levels of neon-21 have been the subject of extensive studies ${ }^{1-8}$ in recent years and the strong coupling collective model with $\mathrm{K}$ band mixing has been used for their interpretation ${ }^{2,4,6,9,10}$. Two states, which are predominantly members of $K=1 / 2$ and $K=3 / 2$ rotational bands and have $J \pi=1 / 2^{+}$and $9 / 2^{+}$respectively, are predicted ${ }^{9,10}$ near to $3 \mathrm{MeV}$ excitation in neon-21. In addition, Freeman ${ }^{10}$ has predicted a $J \pi=5 / 2^{+}$state near $3 \mathrm{MeV}$ and a $3 / 2^{+}$state near $3.5 \mathrm{MeV}$, while Davidson ${ }^{9}$ shows three states of $3 / 2^{+}, 1 / 2^{+}$and $5 / 2^{+}$between 3.5 and $4 \mathrm{MeV}$. In this calculation an additional $\mathrm{K}=1 / 2$ band, based on Nilsson orbit 6 , was included.

Recent high-resolution $\gamma$-ray studies ${ }^{7,8}$, using the reactions ${ }^{18} \mathrm{O}(\alpha, n \gamma)^{21} \mathrm{Ne}$ and ${ }^{20} \mathrm{Ne}(\mathrm{d}, \mathrm{p} \gamma)^{21} \mathrm{Ne}$, have established a doublet in neon-21 at 2.791 and $2.797 \mathrm{MeV}$. The latter state has been shown ${ }^{8,11}$ to be $J \pi=1 / 2^{+}$as the former is not populated in the reaction ${ }^{20} \mathrm{Ne}(\mathrm{d}, \mathrm{pr})^{21} \mathrm{Ne} . \quad \mathrm{J}^{\pi}=1 / 2^{+}$ was also suggested for the $2 \cdot 791 \mathrm{MeV}$ state by Pelte et al. ${ }^{4}$ from an application of the $(2 J+1)$ rule to the averaged ${ }^{23} \mathrm{Na}(\mathrm{d}, \alpha)^{21} \mathrm{Ne}$ cross-sections. He subsequently explained ${ }^{12}$ the reduced M1 transition probabilities from the 2.791 state by accepting this assignment which was based on a hole in Nilsson orbit 6 . Bent et al. ${ }^{6}$ were unable to explain this hindrance on the assumption of $5 / 2^{+}$. 